\title{
A tunable Josephson platform to explore many-body quantum optics in circuit-QED
}

Javier Puertas Martínez ${ }^{1}$, Sébastien Léger ${ }^{1}$, Nicolas Gheeraert ${ }^{1}$, Rémy Dassonneville ${ }^{1}$, Luca Planat ${ }^{1}$, Farshad Foroughi ${ }^{1}$, Yuriy Krupko ${ }^{1}$, Olivier Buisson ${ }^{1}$, Cécile Naud ${ }^{1}$, Wiebke Hasch-Guichard ${ }^{1}$, Serge Florens ${ }^{1}$, Izak Snyman ${ }^{2}$ and Nicolas Roch ${ }^{1}$

The interaction between light and matter remains a central topic in modern physics despite decades of intensive research. Coupling an isolated emitter to a single mode of the electromagnetic field is now routinely achieved in the laboratory, and standard quantum optics provides a complete toolbox for describing such a setup. Current efforts aim to go further and explore the coherent dynamics of systems containing an emitter coupled to several electromagnetic degrees of freedom. Recently, ultrastrong coupling to a transmission line has been achieved where the emitter resonance broadens to a significant fraction of its frequency, and hybridizes with a continuum of electromagnetic (EM) modes. In this work we gain significantly improved control over this regime. We do so by combining the simplicity and robustness of a transmon qubit and a bespoke EM environment with a high density of discrete modes, hosted inside a superconducting metamaterial. This produces a unique device in which the hybridisation between the qubit and many modes (up to ten in the current device) of its environment can be monitored directly. Moreover the frequency and broadening of the qubit resonance can be tuned independently of each other in situ. We experimentally demonstrate that our device combines this tunability with ultrastrong coupling and a qubit nonlinearity comparable to the other relevant energy scales in the system. We also develop a quantitative theoretical description that does not contain any phenomenological parameters and that accurately takes into account vacuum fluctuations of our large scale quantum circuit in the regime of ultrastrong coupling and intermediate non-linearity. The demonstration of this new platform combined with a quantitative modelling brings closer the prospect of experimentally studying many-body effects in quantum optics. A limitation of the current device is the intermediate nonlinearity of the qubit. Pushing it further will induce fully developed many-body effects, such as a giant Lamb shift or nonclassical states of multimode optical fields. Observing such effects would establish interesting links between quantum optics and the physics of quantum impurities

npj Quantum Information (2019)5:19; https://doi.org/10.1038/s41534-018-0104-0

\section{INTRODUCTION}

Due to strong interactions between elementary constituants, correlated solids ${ }^{1}$ and trapped cold atoms ${ }^{2}$ host fascinating manybody phenomena. Attempts to produce similar effects in purely optical systems are hampered by the obvious fact that photons do not naturally interact with each other. If this obstacle can be overcome, there is the tantalizing prospect of probing the manybody problem using the contents of the quantum optics toolbox, such as single photon sources and detectors, high-order correlations in time-resolved measurements, entanglement measures, and phase space tomographies to name a few ${ }^{3}$.

One route to building a many-body quantum optical system is to rely on arrays of strongly non-linear cavities or resonators ${ }^{4,5,6}$, but minimising disorder in such architectures is a formidable challenge. Another route that circumvents these difficulties involves coupling a single well-controlled non-linear element to a disorder free harmonic environment ${ }^{7-11}$. If the difficult experimental challenge of engineering an ultra-strong coupling can be overcome ${ }^{12-17}$, thus exceeding the boundaries of the standard single photon regime in quantum optics, this approach could pave the way to bosonic realizations of electronic impurity systems such as the famous Kondo and Anderson models ${ }^{4,18-23}$.
Our goal here is to achieve a large coupling between a sufficiently non-linear qubit and a quantum coherent environment containing many harmonic degrees of freedom ${ }^{24-26}$.

When coupling an impurity to a finite size electromagnetic environment, five important frequency scales have to be considered. The impurity is characterized by its qubit frequency $\omega_{\text {qubit, }}$ i.e., the excitation frequency between its two lowest internal states. Real impurities always possess more than two levels. The anharmonicity $a$, defined as the difference between $\omega_{\text {qubit }}$ and the frequency for excitation from the second to third internal impurity state, characterises the departure of an impurity from a trivial harmonic oscillator $(a \rightarrow 0)$ or a pure two-level system $(a \rightarrow \infty)$. The coupling between the impurity and environment is characterized by the spontaneous emission rate $\Gamma$ at which the impurity exchanges energy with its environment. The environment itself is characterized by its free spectral range $\delta \omega$, which measures the typical frequency spacing between environmental modes, and the spectral broadening $k$ of these modes due to their coupling to uncontrolled degrees of freedom. The soughtafter multi-mode regime is obtained when $\Gamma$ is larger than $\delta \omega$ so that the impurity is always coupled to several discrete environmental modes, producing a cluster of hybridized qubit-

\footnotetext{
'Univ. Grenoble Alpes, CNRS, Grenoble INP, Institut Néel, 38000 Grenoble, France and ${ }^{2}$ Mandelstam Institute for Theoretical Physics, School of Physics, University of the Witwatersrand, Johannesburg, South Africa

Correspondence: Nicolas Roch (nicolas.roch@neel.cnrs.fr)
}

Received: 2 September 2018 Accepted: 26 September 2018

Published online: 25 February 2019 

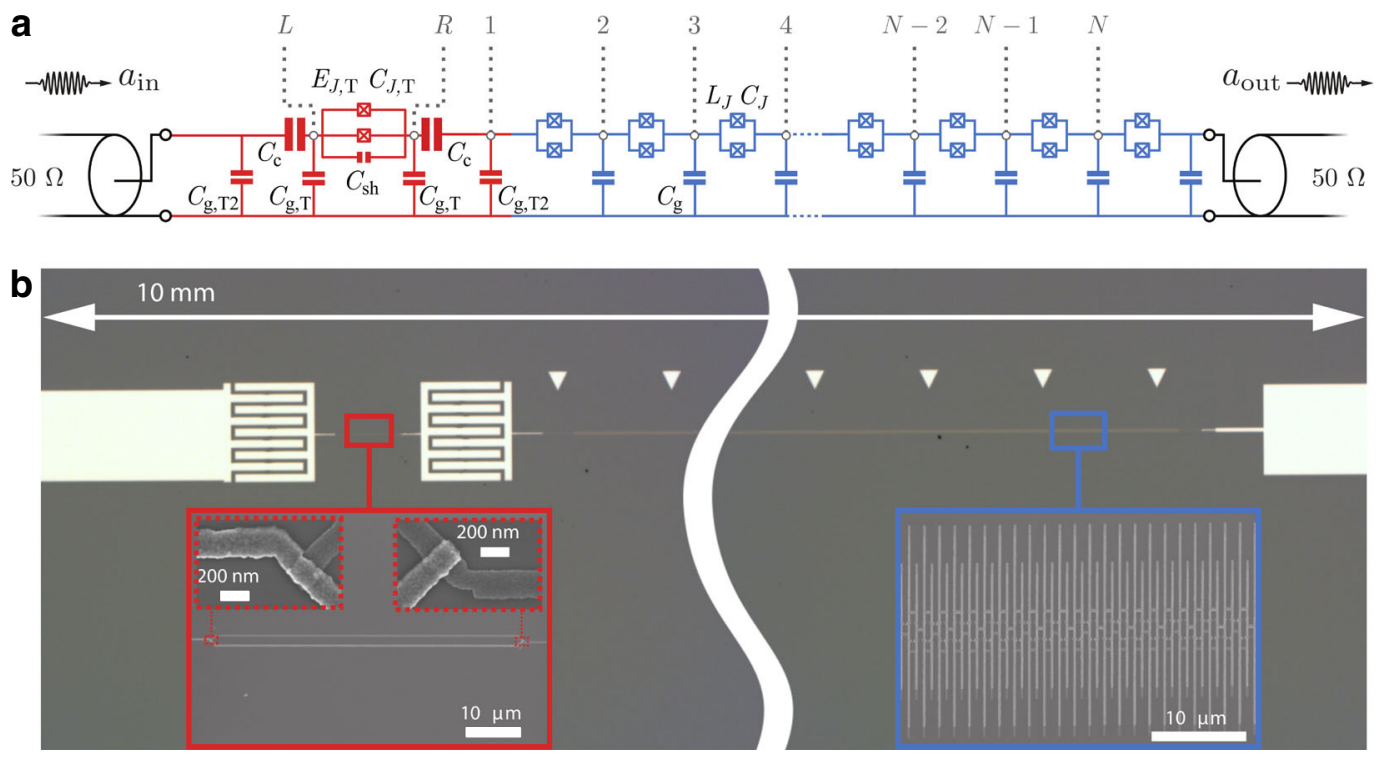

Fig. 1 A Josephson platform for waveguide quantum electrodynamics. a Lumped-element model of the circuit including the nodes used in the calculations. b Optical microscope image of the sample. The two zoom-in are SEM pictures of the SQUID of the qubit (red square) and the SQUIDs in the chain (blue square). The qubit is capacitively coupled to the chain and to a $50 \Omega$ measurement line, via large interdigital contacts. Only a small portion of the Josephson chain, which comprises 4700 SQUIDs in total, is shown

environment resonances ${ }^{27}$. There are several requirements for reaching the many-body regime. First, $\Gamma$ must be a significant fraction of $\omega_{\text {qubit }}$ (ultra-strong coupling). This is a prerequisite for multiparticle decay ${ }^{19,22}$. If the coupling is too weak, the system becomes trivial, since only number-conserving processes are relevant (Equivalently Markov and rotating wave approximations apply.) A second requirement is agt; rsim $\Gamma$. If this condition is not met, the non-linearity of the impurity is swamped by the broadening of the impurity levels, and the same frequency will drive transitions between several impurity levels, so that the system as a whole behaves more like a set of coupled harmonic oscillators than like a two-level system coupled to an environment $^{28}$. Within the many-body regime, two limits can be distinguished. In the case of a finite-size environment that we address here (namely, $\delta \omega>\kappa$ ), each mode of the system can be addressed and controlled individually, while in the limit of a thermodynamically large environment $(\delta \omega / \kappa \rightarrow 0)$ one recovers a smooth dissipation-broadened qubit resonance.

The many-body ultra-strong coupling regime (defined by the first two conditions above) is hard to reach in quantum optics experiments because the coupling to three-dimensional vacuum fluctuations arises at order $\left[a_{\mathrm{QED}}\right]^{3}$, with $a_{\mathrm{QED}} \simeq 1 / 137$ the fine structure constant ${ }^{3}$. However, for superconducting qubits coupled to transmission lines, the scaling is much more favorable ${ }^{20,29-31}$ than in a vacuum. Indeed the ratio $\Gamma / \omega_{\text {qubit }}$ can essentially be made arbitrarily large, provided the impedance of the environment matches that of the qubit (see Sec. E of the Supplementary Information). Building on this ability of superconducting circuits to reach very large couplings, several experiments demonstrated the ultra-strong coupling regime in coupled qubit/cavity systems ${ }^{14-16,32}$. The rich physics associated to this coupling regime has also been evidenced using quantum simulation ${ }^{33,34}$. The condition $\Gamma>\delta \omega$ has also been fulfilled by coupling superconducting qubits to open transmission lines ${ }^{35-37}$ or engineered resonators ${ }^{7,9}$. However, it is only recently that the necessary conditions for the many-body regime were demonstrated concurrently ${ }^{12,13}$. The device of ${ }^{12,13}$ consists of a flux qubit coupled to the continuum provided by a superconducting transmission line, which realises the thermodynamic limit $(\delta \omega /$ $\kappa \rightarrow 0$ ). Limitations of such setups include the lack of a microscopic model (since it is hard to characterize the waveguide properties of a transmission line outside the relatively narrow $4-8 \mathrm{GHz}$ band where microwave transmission experiments can comfortably be performed), and importantly, that the transmission line is not an in situ tunable environment.

\section{RESULTS AND DISCUSSION}

In this work, we circumvent the above limitations, by designing circuits that provide independent tunability of both a qubit and a finite size but very large environment, while allowing highprecision spectroscopic measurements of the environment itself $(\delta \omega>\kappa)$ and first principle modeling. Our device, shown in Fig. $1 \mathrm{~b}$, consists of a transmon qubit, which is relatively insensitive to both charge and flux noise, capacitively coupled to a long onedimensional Josephson metamaterial, comprising 4700 SQUIDs. Such chains have been studied since the early 90 's in the context of the superconductor-insulator transition ${ }^{38-41}$ or to explore dual of the Josephson effect ${ }^{42-44}$. Our setup differs in two ways from these previous works. First, we took great care to produce a chain in the linear regime, far from the onset of non-linear effects such as quantum phase slips ${ }^{45}$, so that one of the basic benefits of quantum optics, i.e., the elimination of non-linearities where they are not wanted, is realized. Second, we performed AC microwave spectroscopy of our device, instead of DC transport measurements. This allows us to characterize the electromagnetic degrees of freedom, also called Mooij-Schön plasma modes ${ }^{28,46-51}$, microscopically. We managed to resolve as many as 50 individual low frequency electromagnetic modes of this non-dissipative and fully tunable environment (see Fig. 2c). An essential property of the Josephson metamaterial is its high characteristic impedance $Z_{c}=\sqrt{L_{J} / C_{g}} \simeq 1590 \Omega$, which being of the same order of magnitude as the effective impedance of our transmon qubit, $Z_{T}=\hbar /(2 e)^{2} \sqrt{2 E_{C, T} / E_{J, T}} \simeq 760 \Omega$ (here at zero flux), allow us to reach multi-mode ultrastrong coupling. The simplicity of the transmon architecture enables us to either compute from first principles or to extract all the parameters necessary to construct a microscopic model of the full system, without dropping the socalled ' $A^{2}$-terms', a routine approximation in optics ${ }^{52-56}$, that however breaks down at ultra-strong coupling.

Our measurements are based on the frequency-resolved microwave transmission through the whole device. Figure $2 b$ 
a

Flux $\left(\Phi_{\mathrm{c}} / \Phi_{0}\right)$

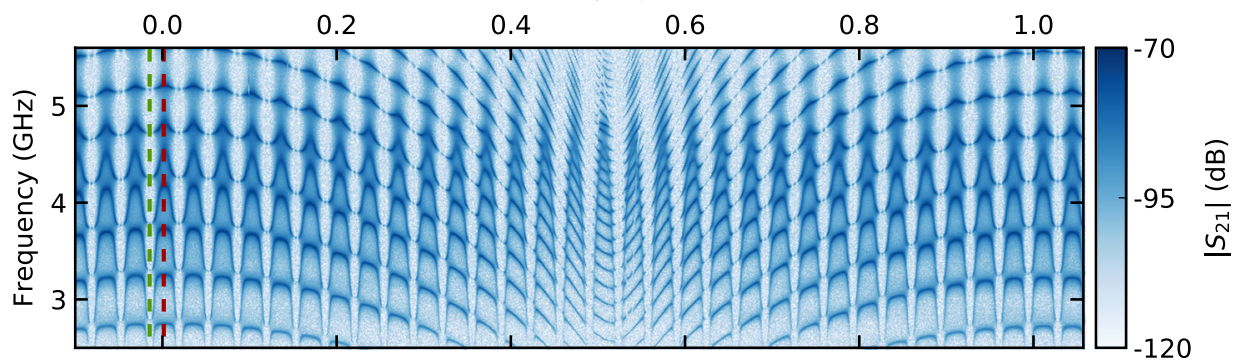

b

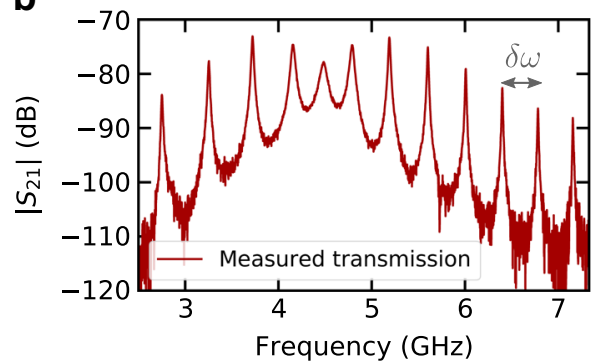

C

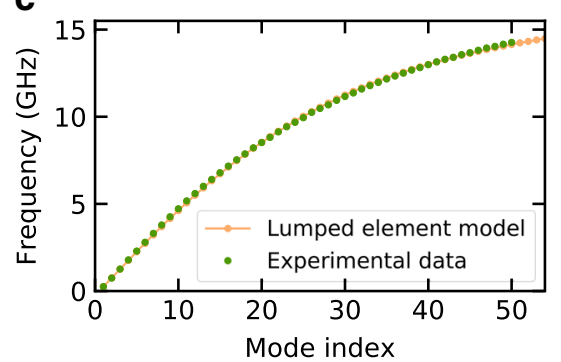

Fig. 2 Spectroscopic analysis of the full quantum circuit. a Microwave transmission measurement of the complete device (transmon and chain) shown in panel $\mathbf{b}$ of Fig. 1, as a function of flux. Two flux periods can be seen, the long one (resp. the short one) being related to the SQUIDs in the chain (resp. in the qubit). Two vertical cuts indicate the spectroscopic traces shown in the two bottom panels respectively. $\mathbf{b}$ Frequency trace of microwave transmission through the device at flux $\Phi_{\mathrm{C}}=0$ (red cut in panel a), in which case the transmon flux is also $\Phi_{\mathrm{T}}=$ 0 . The free spectral range $\delta \omega$ is shown in grey. c Dispersion relation of the chain alone obtained from the fit of the resonances at chain flux $\Phi_{C}$ $=-0.015 \Phi_{0}$ corresponding to $\Phi_{\mathrm{T}}=-\Phi_{0} / 2$ (green cut in panel a), so that the chain modes do not hybridize with the transmon

shows the amplitude of the transmitted field at a fixed value of the external magnetic field, and at low probe power. This spectrum reveals a set of resonances in our device, displaying a narrow spectral broadening $k / 2 \pi=20 \mathrm{MHz}$ (for the non hybridized modes of the chain) caused by the coupling to the $50 \Omega$ contacts. As the external magnetic field is varied, two modulation periods are seen in the resonance spectrum (Fig. 2a). The short and long periods correspond respectively to a one quantum $\Phi_{0}=h / 2 e$ increase of the flux through the large transmon or through the small chain SQUID loops. This feature allows us to adjust independently the flux threading the transmon SQUID loop $\left(\Phi_{T}\right)$ from the one threading the chain SQUID loops $\left(\Phi_{C}\right)$. The former controls the qubit frequency, while the latter controls the impedance of the environment, and hence the qubitenvironment coupling strength. Before studying the hybridization between the transmon and chain, we characterize the chain on its own by setting $\Phi_{\mathrm{T}}=\Phi_{0} / 2$, so that the qubit decouples from it (See Fig. $2 \mathrm{c}$ and Sec. B of the Supplementary Information). We obtain a good fit between the dispersion relation predicted by a microscopic model and the one extracted from the measured resonances. This allows us to extract all of the parameters necessary to characterize the chain modes.

The transmon qubit becomes active when $\Phi_{\mathrm{T}} \neq \Phi_{0} / 2$, and is expected to hybridize with the chain. Figure $3 a$ shows a lowpower spectroscopy of the system as a function of $\Phi_{\mathrm{T}}$, keeping $\Phi_{\mathrm{C}}$ nearly constant. When a chain mode is not hybridized with the qubit, the corresponding spectral line runs nearly horizontally. When $\Phi_{T}$ is varied, the qubit frequency sweeps across the resonances of the chain modes and creates a clear pattern of several avoided level crossings (See Fig. 3a). We note that at fixed $\Phi_{\mathrm{T}}$, several chain modes in the vicinity of the transmon resonance are visibly displaced. Thus, the transmon simultaneously hybridizes with many modes. This is a signature of multi-mode ultrastrong coupling, a topic that will be further addressed below. Evidence that the transmon behaves as a qubit is provided by its saturation spectrum (Fig. 3b). Here the fluxes $\Phi_{\mathrm{T}}$ and $\Phi_{\mathrm{C}}$ are kept constant, while the transmission through the system is recorded at increasing probe power. For a harmonic system, the resonance positions are independent of driving power. In an anharmonically oscillating classical system, a gradual dependence on the driving power would appear. Experimentally, we observe that below a driving power $\sim-10 \mathrm{dBm}$, the resonance positions in the transmission spectrum are independent of the driving probe power. As the driving power increases beyond $-10 \mathrm{dBm}$, the peak around $4.5 \mathrm{GHz}$ disappears while the other peaks assume the positions they have when the qubit is inactive. (The horizontal lines correspond to the low power spectrum when $\Phi_{\mathrm{T}}=\Phi_{0} / 2$.) This is evidence of saturation, a clear qubit signature ${ }^{18,35,36}$. The fact that several chain modes are shifted when the transmon is saturated constitutes an additional proof that the transmon hybridizes with many modes at once.

To quantify the hybridization of the transmon mode with the chain modes, we compare the normal mode spectrum of the full system at $\Phi_{\mathrm{T}} \neq \Phi_{0} / 2$ to the spectrum at $\Phi_{\mathrm{T}}=\Phi_{0} / 2$. As mentioned above, in the latter case, the transmon decouples from the bare modes of the chain. The system with $\Phi_{\mathrm{T}} \neq \Phi_{0} / 2$ therefore has one extra mode in the vicinity of the transmon frequency. We define the relative frequency shift $\delta \phi_{n}\left(\Phi_{T}, \Phi_{C}\right)$ as the difference in frequency between the $n$th mode of the coupled and uncoupled chains, normalized to the free spectral range of the chain $\delta \omega_{n}\left(\Phi_{\mathrm{T}}\right.$, $\left.\Phi_{C}\right)=\omega_{n}\left(\Phi_{0} / 2, \Phi_{C}\right)-\omega_{n-1}\left(\Phi_{0} / 2, \Phi_{C}\right)$, and including a $\pi$ factor for later convenience, i.e.,

$\delta \phi_{n}\left(\Phi_{\mathrm{T}}, \Phi_{\mathrm{C}}\right)=\pi \frac{\omega_{n}\left(\Phi_{0} / 2, \Phi_{\mathrm{C}}\right)-\omega_{n}\left(\Phi_{\mathrm{T}}, \Phi_{\mathrm{C}}\right)}{\delta \omega_{n}\left(\Phi_{\mathrm{T}}, \Phi_{\mathrm{C}}\right)}$

where $\omega_{n}\left(\Phi_{\mathrm{T}}, \Phi_{C}\right)$ is the frequency of the $n$th lowest non-zero mode for a given flux in the transmon and in the chain. This frequency shift is readily extracted from the peak positions in our global spectroscopic map (Fig. 2a). Remarkably (see Sec. $\mathrm{H}$ of the Supplementary Information for a derivation), $\delta \phi_{n}\left(\Phi_{\mathrm{T}}, \Phi_{\mathrm{C}}\right)$ in Eq. (1) equals the phase shift experienced by mode $n$ due to the presence of the nearby transmon mode:

$\delta \phi_{n}\left(\Phi_{\mathrm{T}}, \Phi_{\mathrm{C}}\right)=\phi_{n}\left(\Phi_{\mathrm{T}}, \Phi_{\mathrm{C}}\right)-\phi_{n}\left(\Phi_{0} / 2, \Phi_{\mathrm{C}}\right)$, 

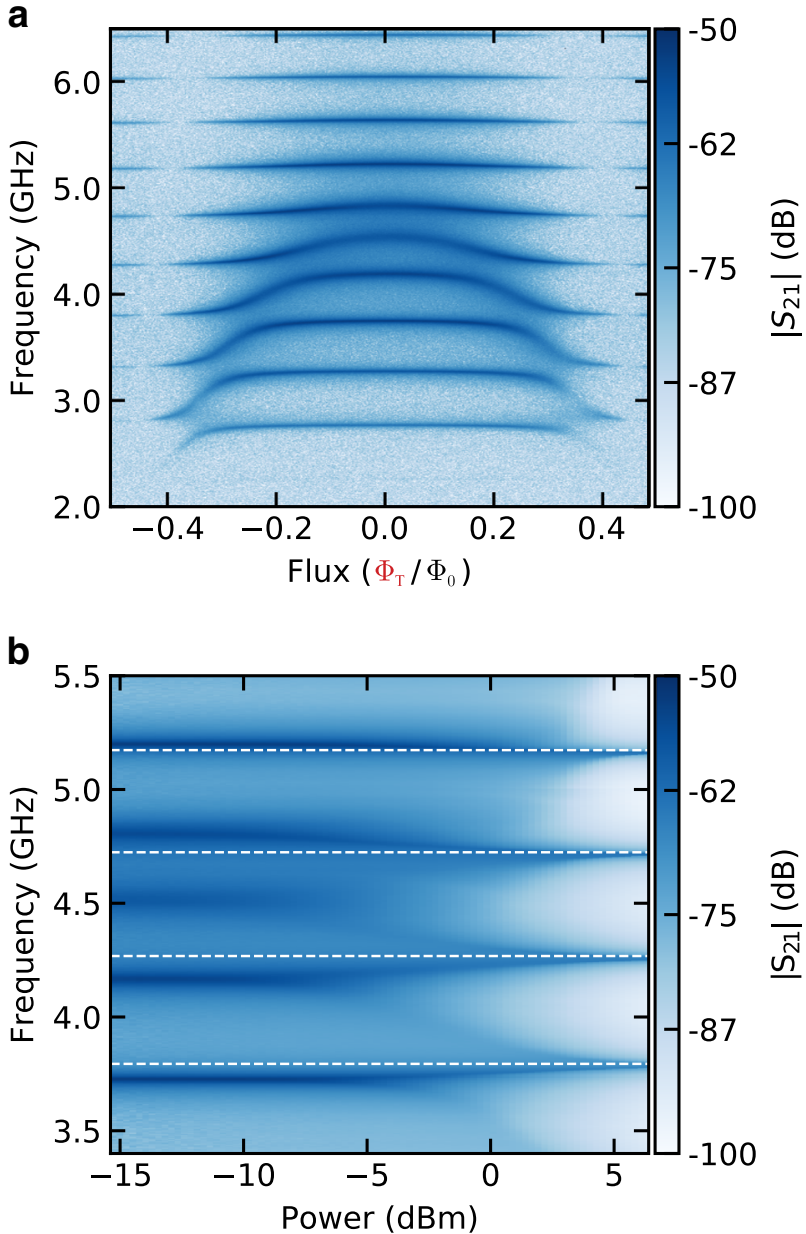

Fig. 3 Hybridization of qubit and chain modes. a Transmission spectrum as a function of transmon flux $\Phi_{T}$, at chain flux $\Phi_{C} \simeq 0$ (representing a small portion of the full spectroscopy in Fig. 2a) The horizontal lines are the chain modes far from the qubit resonance. The flux modulation of the transmon frequency produces a bellshaped succession of anticrossings. b Transmission spectrum as a function of applied microwave power at fixed fluxes $\Phi_{T}=\Phi_{\mathrm{C}}=0$. The white dashed lines indicate the modes of the array at $\Phi_{\mathrm{T}}=\Phi_{0} / 2$. With increasing power, the transmon-like mode near 4.5 disappears, showing its non-linear quantum character. In addition the modes of the array shift to their bare frequencies

where $\phi_{n}\left(\Phi_{T}, \Phi_{C}\right)$ is the phase shift of mode $n$ of the full system at transmon flux $\Phi_{\mathrm{T}}$ and chain flux $\Phi_{\mathrm{C}}$. From Eq. (1) it follows that the phase shift equals 0 (resp. $\pi$ ) for modes far below (resp. far above) the renormalized transmon frequency. For hybridized modes in the vicinity of the transmon line, $\delta \phi_{n}\left(\Phi_{\mathrm{T}}, \Phi_{\mathrm{C}}\right)$ lies between 0 and $\pi$. This behavior is clearly observed in Fig. 4a where the measured relative frequency shifts are reported for a chain flux $\Phi_{C}=0$ and various transmon fluxes $\Phi_{\mathrm{T}}$. The wide frequency dispersion of intermediate $\delta \phi_{n}\left(\Phi_{\mathrm{T}}, \Phi_{\mathrm{C}}\right)$ provides direct evidence for a hybridization with up to ten chain modes. In the thermodynamic limit of an infinite chain with perfect impedance matching to the measurement ports, the transmon-induced phase shift $\delta \phi_{n}\left(\Phi_{T}, \Phi_{C}\right)$ becomes a continuous function $\delta \phi\left(\omega, \Phi_{\mathrm{T}}, \Phi_{\mathrm{C}}\right)$ of the mode frequency $\omega$. Moreover, it can be shown that the frequency derivative of $\delta \phi\left(\omega, \Phi_{\mathrm{T}}, \Phi_{\mathrm{C}}\right)$ matches very precisely the theoretically expected lineshape of the dissipative response of the transmon coupled to an infinite environment. (See Sec. I of the Supplementary Information). This constitutes a central finding of our work: the renormalized transmon frequency $\omega_{\mathrm{T}}$ and linewidth $\Gamma_{\mathrm{T}}$ can be directly inferred from a measurement of the phase shifts of the
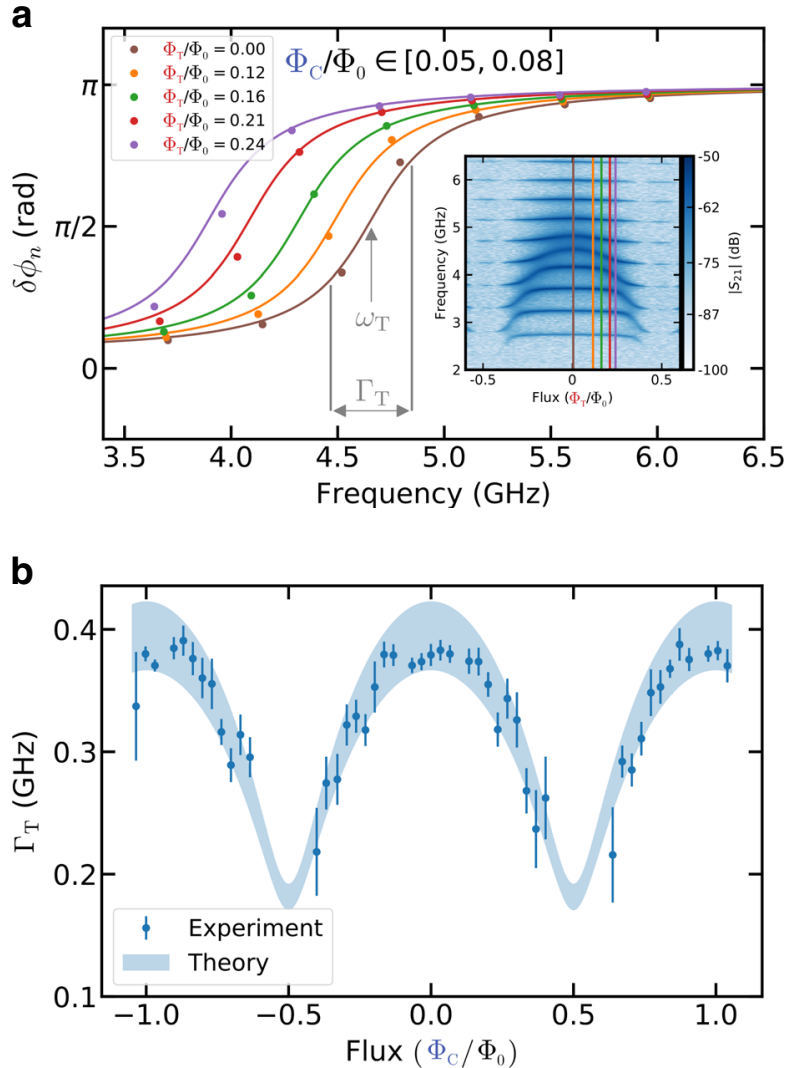

Fig. 4 Extraction of qubit properties from the measurement of its controlled environment. a Phase shift $\delta \phi_{n}$ of the discrete chain modes as a function of mode frequency $\omega_{n}$ for different transmon fluxes $\Phi_{\mathrm{T}}$, fixing $\Phi_{\mathrm{C}}=0$. The solid line is a fit using Eq. (2). The inset shows the chosen transmon fluxes $\Phi_{T}$ as line cuts in the transmission measurement (with the same color code). b The transmon width $\Gamma_{\mathrm{T}}$ for different fluxes in the chain $\Phi_{\mathrm{C}}$, showing control of the coupling to the large but discrete environment. The experimental points (dots) are obtained from an arctangent fit of the data in panel a. For better visibility, only the flux values where the transmon frequency is maximum are included. The blue shaded area represents the theoretical expectation for $\Gamma_{\mathrm{T}}$, within a confidence interval given by the error in the capacitances in Table 1

individual modes in the finite bath. In terms of measurement protocol however, there is a sharp difference between the chain mode phase shifts and the qubit response functions. Usually, the qubit response is obtained by observing the transmon, and its environment can be viewed as a black box that combines unmonitored decoherence channels as well as the physical ports used for measurement. This procedure constitutes the usual paradigm in the study of open quantum systems. Our protocol is unusual because information about an open quantum system is obtained by monitoring the discrete modes that constitute its dominant environment.

We finally turn to a quantitative analysis of our data, including a comparison to the predictions of a microscopic model, in order to determine if the requirements for reaching the many-body regime has been met. By extracting the maximum renormalized transmon frequency $\omega_{T \text {,max }}$ extracted from the phase shift data of Fig. $4 a$ at chain flux $\Phi_{\mathrm{T}}$ an integer multiples of $\Phi_{0}$, we are able to infer the only remaining unknown system parameter, namely the maximum transmon Josehpson energy $E_{J, \mathrm{~T}, \max }$. This allows us to estimate the anharmonicity of the transmon $a$, which ranges from $0.36 \mathrm{GHz}$ at $\Phi_{\mathrm{T}}=0$ to $0.44 \mathrm{GHz}$ at $\Phi_{\mathrm{T}}=0.3 \Phi_{0}$. We emphasize that the condition agt; $r \operatorname{sim} \Gamma_{\mathrm{T}}$ for anharmonic many-body behavior is 
thus fulfilled (see Fig. $4 \mathrm{~b}$ for the extracted transmon linewidth $\Gamma_{\mathrm{T}}$ which lies in the range $0.2-0.4 \mathrm{GHz}$ ). Using the extracted parameters to calculate $\delta \phi\left(\omega, \Phi_{\mathrm{T}}, \Phi_{\mathrm{C}}\right)$ according to Eq. (11) we find the predicted theoretical lines in Fig. 4a. The excellent agreement between theory and experiment seen here for six different values of $\Phi_{\mathrm{T}}$ persists for each of the hundreds of $\left(\Phi_{\mathrm{T}}, \Phi_{\mathrm{C}}\right)$ combinations where we have made the comparison. (See Sec. C of the Supplementary Material for a further selection of results.) We stress that this agreement is obtained after all model parameters have been fixed, so that there is no fitting involved in comparing the predicted and measured phase shifts. The quantitative modeling of such a large quantum circuit clearly is an important landmark in the field of open quantum systems. In Fig. 4b we examine the transmon linewidth $\Gamma_{T}$ that we extracted from the phase shift data, as a function of chain flux $\Phi_{C}$ for fixed transmon flux $\Phi_{T}=0$. Very good agreement (with no fitting parameters) is again obtained with the prediction of our model. These results demonstrate that we can tune the qubit-environment coupling independently from $\omega_{\mathrm{T}}$ using the flux in the chain, and that we achieved the ultra-strong coupling in our waveguide, i.e., coupling to a large number (here 10) of modes with a sizeable linewidth $\Gamma_{\mathrm{T}} / \omega_{\mathrm{T}} \simeq 0.1$. A hallmark of ultra-strong coupling is the failure of the rotating wave approximation (RWA), as previously discussed in coupled qubit and cavity systems ${ }^{14}$. We have examined the consequences of the RWA on our microscopic model (see Sec. J of the Supplementary Information), and found a discrepancy of $100 \mathrm{MHz}$ in the transmon frequency $\omega_{\mathrm{T}}$, showing the quantitative importance of non-RWA terms. We would like to stress that demonstrating the relevance of these terms is much more than obtaining a good data-theory agreement. With counter-rotating contributions in the few percent range, we expect a finite rate for parametric processes in which photon-number is not conserved. In future we plan to use the current platform to observe these interesting many-body effects directly.

In conclusion, this work provides the first demonstration of many-body ultra-strong coupling between a transmon qubit and a large and tunable bath. To obtain full control over the environment, a superconducting metamaterial, comprising 4700 SQUIDs, was employed. Although this quantum circuit contains a huge number of degrees of freedom, we were able to characterise all its parameters in situ. This allows us to demonstrate unambiguously that our systems meets the three conditions required to reach the many-body regime, namely $\Gamma g t ; r \operatorname{sim} \delta \omega$, $\Gamma g t ; r \operatorname{sim} 0.1 \omega_{\text {qubit, }}$ and agt; $r \operatorname{sim} \Gamma$. A novel experimental methodology was implemented to analyze the qubit properties by means of the extraction of the phase shifts of the environmental modes. Despite the large size of our quantum circuit, we succeeded in providing a fully microscopic model which accounts for the transmon response without any fitting parameters. We also found that the qubit linewidth for the long chain agreed with results in the thermodynamic limit, showing that the finite environment has the same influence on the qubit as a truly macroscopic bath. The further possibility to tune the coupling to the environment in situ, demonstrated by a $50 \%$ flux-modulation of the qubit linewidth, opens the way to controlled quantum optics experiments where many-body effects are fully-developed ${ }^{18,19,21,22,57,58}$, as well as more advanced environmental engineering for superconducting qubits 5 .

\section{METHODS}

Sample fabrication and parameters

The sample was fabricated on a highly resistive silicon substrate, using a microstrip geometry. The ground is defined as the backside of the wafer which was gold-plated, ensuring a good electrical conductivity. Interdigital capacitances were chosen to connect the transmon and the metamaterial. They do not provide the lowest surface participation factor ${ }^{59,60}$ but they allow us to maximize the coupling capacitances $C_{c}$ of the transmon to the
Table 1. Sample parameters

\begin{tabular}{lllc}
\hline Chain parameters & & \multicolumn{3}{c}{ Transmon qubit parameters } \\
\hline$L_{J, \text { min }}$ & $(0.33 \pm 0.02) \mathrm{nH}$ & $C_{\mathrm{g}, \mathrm{T} 2}$ & $(33 \pm 1) \mathrm{fF}$ \\
$C_{\mathrm{g}}$ & $(0.13 \pm 0.01) \mathrm{fF}$ & $C_{\mathrm{g}, \mathrm{T}}$ & $(48 \pm 2) \mathrm{fF}$ \\
$C_{J}$ & $(259 \pm 14) \mathrm{fF}$ & $C_{\mathrm{C}}$ & $(119 \pm 2) \mathrm{fF}$ \\
$N$ & 4700 & $C_{\mathrm{sh}}$ & $(6.9 \pm 0.1) \mathrm{fF}$ \\
$d$ (asymmetry) & 0.25 & $C_{J, \mathrm{~T}}$ & $(5.2 \pm 0.3) \mathrm{fF}$ \\
& & $E_{J, \mathrm{~T}, \max } / h$ & $(10.2 \pm 0.4) \mathrm{GHz}$ \\
& & $E_{\mathrm{C}, \mathrm{T}} / h$ & $(2.4 \pm 0.1) \mathrm{GHz}$ \\
\hline
\end{tabular}

chain, while minimizing the capacitances to ground $C_{\mathrm{g}, \mathrm{T}}$ and $C_{\mathrm{g}, \mathrm{T} 2}$. (See panel a of Fig. 1 for the definitions of the these capacitances.) This system is probed via two $50 \Omega$ transmission lines, one of which is capacitively coupled to the transmon, while the other is galvanically coupled to the chain. The whole device (Josephson junctions, capacitances and transmission lines) was fabricated in a single electron-beam lithography step, using a bridge-free fabrication technique ${ }^{61}$. The Josephson elements of the chain are tailored to be deep in the linear regime $\left(E_{J} / E_{C}=8400\right)$, where $E_{J}$ and $E_{C}$ are the respectively the Josephson energy at $\Phi_{C}=0$ and the charging energy of a chain element), leaving the transmon as the main source of non-linearity in the system. All parameters of the system are listed in Table 1. Here, $L_{J \text {,min }}$ is the minimum inductance of a chain SQUID loop, (which occurs when $\Phi_{C}=0$ ). There is a slight asymmetry between the two Josephson junctions that constitute a single chain SQUID loop, which is quantified by the asymmetry parameter $d$. The flux-dependent inductance $L_{J}\left(\Phi_{C}\right)$ of a chain SQUID loop is given by

$$
L_{J}\left(\Phi_{\mathrm{C}}\right)=\frac{L_{J, \text { min }}}{\sqrt{\cos ^{2}\left(\pi \Phi_{C} / \Phi_{0}\right)+d^{2} \sin ^{2}\left(\pi \Phi_{C} / \Phi_{0}\right)}},
$$

and the corresponding Josephson energy by $E_{J}\left(\Phi_{C}\right)=\varphi_{0}^{2} / L_{J}\left(\Phi_{C}\right)$ with $\varphi_{0}$ $=\hbar / 2 e$ the reduced flux quantum. The transmon Josephson junctions are symmetric, and hence the flux dependent transmon Josephson energy is given by

$$
E_{J, \mathrm{~T}}\left(\Phi_{\mathrm{T}}\right)=E_{J, \mathrm{~T}, \max }\left|\cos \left(\pi \Phi_{\mathrm{T}} / \Phi_{0}\right)\right|
$$

The transmon charging energy is not an independent parameter (see Eq (9)), but is listed in the table, due to the prominent role it plays in what follows. The meanings of the remaining parameters in Table 1 are explained in panel a of Fig. 1. The majority of the parameter values listed are obtained either using a finite-element solver (Sonnet) or extracted from the measured dispersion relation of the chain at $\Phi_{\mathrm{T}}=0$. (See Sec. B and D of the suplementary Material.) The only exceptions are $C_{J}$, that is obtained from knowledge of the junction areas via an empirical formula, and $E_{J, T, m a x}$ that we extract via a procedure described in the last subsection below, which uses the data at $\Phi_{T}$ equal to multiples of $\Phi_{0}$ in panel a of Fig. 2.

\section{Full model}

The circuit diagram for the lumped-element model is shown in Fig. 1. It consists of $N+2$ nodes, where $N$ is the number of SQUIDs in the chain. To describe the circuit, we use the Cooper pair number operator $\hat{n}_{j}$, which gives the number of Cooper pairs in node $j$, and the superconducting phase operator $\hat{\varphi}_{j}$, which gives the superconducting phase at node $j$. They satisfy the canonical commutation relations $\left[\hat{n}_{j}, \hat{\varphi}_{l}\right]=i \delta_{j l}{ }^{62}$. Here $j, l \in[L, R$, $1,2,3, \ldots, N]$ with $L$ and $R$ referring to the left and right transmon nodes. As explained before, the SQUIDs of the chain are linear inductors, to a very good approximation. We define $\hat{n}^{T}=\left(\hat{n}_{L}, \hat{n}_{R}, \hat{n}_{1}, \ldots, \hat{n}_{N}\right)$ and $\hat{\varphi}^{T}=\left(\hat{\varphi}_{L}, \hat{\varphi}_{R}, \hat{\varphi}_{1}, \ldots, \hat{\varphi}_{N}\right)$. In this notation, the Hamiltonian of the circuit is given by

$H=\frac{(2 e)^{2}}{2} \hat{n}^{T} \hat{C}^{-1} \hat{n}-\frac{1}{2} \hat{\varphi}^{T} \hat{\jmath} \hat{\varphi}-E_{J, T}\left(\Phi_{T}\right) \cos \left(\hat{\varphi}_{R}-\hat{\varphi}_{L}\right)$

$\hat{C}$ is the capacitance matrix, such that elements $[\hat{C}]_{j l}=[\hat{C}]_{l j}$ equal the capacitive coupling between the charges on islands $j$ and $l$. In the same way, elements $[\hat{\jmath}]_{j l}=[\hat{\jmath}]_{l j}$ of matrix $\hat{\jmath}$ contains the Josephson energy that couples the superconducting phase on island $j$ and island $I$. Both matrices are $(N+2) \times(N+2)$. Their explicit forms are given below in Eqs. (6) and (7). In both matrices, the boundary conditions that determine entries $(L, L)$ and $(N, N)$ are obtained by assuming that the nodes to the left of node $L$ and to 
the right of node $N$ are grounded.

$\hat{C}=\left(\begin{array}{cccccccc}C_{0} & -C_{\mathrm{sh}, \mathrm{T}} & 0 & 0 & 0 & 0 & \cdots & 0 \\ -C_{\mathrm{sh}, \mathrm{T}} & C_{0} & -C_{\mathrm{c}} & 0 & 0 & 0 & \cdots & 0 \\ 0 & -C_{\mathrm{c}} & C_{1} & -C_{J} & 0 & 0 & \ldots & 0 \\ 0 & 0 & -C_{J} & C_{\Sigma} & -C_{J} & 0 & \ldots & 0 \\ \vdots & \vdots & \vdots & \ddots & \ddots & \ddots & \ldots & 0 \\ 0 & 0 & 0 & 0 & -C_{J} & C_{\Sigma} & -C_{J} & 0 \\ 0 & 0 & 0 & 0 & 0 & -C_{J} & C_{\Sigma} & -C_{J} \\ 0 & 0 & 0 & 0 & 0 & 0 & -C_{J} & C_{\Sigma}\end{array}\right)$

The elements in the capacitance matrix are given by

$$
\begin{gathered}
C_{0}=C_{\mathrm{c}}+C_{\mathrm{sh}, \mathrm{T}}+C_{\mathrm{g}, \mathrm{T}} \\
C_{1}=C_{\mathrm{c}}+C_{J}+C_{\mathrm{g}, \mathrm{T} 2} \\
C_{\Sigma}=2 C_{J}+C_{\mathrm{g}} \\
C_{\mathrm{sh}, \mathrm{T}}=C_{J, \mathrm{~T}}+C_{\mathrm{sh}}
\end{gathered}
$$$$
\hat{\jmath}=\frac{\varphi_{0}^{2}}{L_{J}\left(\Phi_{C}\right)}\left(\begin{array}{cccccccc}
0 & 0 & 0 & 0 & 0 & 0 & \cdots & 0 \\
0 & 0 & 0 & 0 & 0 & 0 & \cdots & 0 \\
0 & 0 & 1 & -1 & 0 & 0 & \cdots & 0 \\
0 & 0 & -1 & 2 & -1 & 0 & \cdots & 0 \\
\vdots & \vdots & \vdots & \ddots & \ddots & \ddots & \cdots & 0 \\
0 & 0 & 0 & 0 & -1 & 2 & -1 & 0 \\
0 & 0 & 0 & 0 & 0 & -1 & 2 & -1 \\
0 & 0 & 0 & 0 & 0 & 0 & -1 & 2
\end{array}\right)
$$

We define the operators $\hat{n}_{\mathrm{T}}=\left(\hat{n}_{R}-\hat{n}_{L}\right) / 2+$ constant and $\hat{\varphi}_{\mathrm{T}}=\left(\hat{\varphi}_{R}-\right.$ $\left.\hat{\varphi}_{L}\right) / 2$ associated with the transmon dynamics. Introducing these operators, and noting that the total transmon charge $\hat{n}_{R}+\hat{n}_{L}$ is concerved, we can rewrite the Hamiltonian as

$$
\begin{gathered}
H=\frac{E_{C, \mathrm{~T}}}{2} \hat{n}_{\mathrm{T}}^{2}-E_{J, \mathrm{~T}}\left(\Phi_{\mathrm{T}}\right) \cos \left(\hat{\varphi}_{\mathrm{T}}\right)+\frac{(2 e)^{2}}{2} \sum_{j l=1}^{N} \hat{n}_{j}\left[\hat{C}^{-1}\right]_{j, l} \hat{n}_{I} \\
+\frac{\varphi_{0}^{2}}{2 L_{j}\left(\Phi_{C}\right)} \sum_{j=1}^{N}\left(\hat{\varphi}_{j+1}-\hat{\varphi}_{j}\right)^{2}+\hat{n}_{\mathrm{T}} \sum_{j=1}^{N} \nu_{j} \hat{n}_{j}
\end{gathered}
$$

where we defined $\hat{\varphi}_{N+1} \equiv 0$. The transmon charging energy $E_{C, T}$ is given by

$E_{C, T}=(2 e)^{2}\left\{\left[\hat{C}^{-1}\right]_{L L}+\left[\hat{C}^{-1}\right]_{R R}-2\left[\hat{C}^{-1}\right]_{L R}\right\}$.

The coupling of $\hat{n}_{\mathrm{T}}$ to the charge on island $j$ is given by

$\nu_{j}=(2 e)^{2}\left\{\left[\hat{\mathrm{C}}^{-1}\right]_{R j}-\left[\hat{\mathrm{C}}^{-1}\right]_{L j}\right\}$.

Chain modes phase shift in the thermodynamic limit

In Fig. 4a we compare the measured relative frequency shift $\delta \phi_{n}\left(\Phi_{\mathrm{T}}, \Phi_{\mathrm{C}}\right)$ to the theoretically predicted transmon phase shift $\delta \phi\left(\omega, \Phi_{T}, \Phi_{C}\right)$ with which it is expected to agree in the thermodynamic limit. Here we provide the analytical formula for the phase shift $\phi\left(\omega, \Phi_{T}, \Phi_{C}\right)$ of a mode with frequency $\omega$. (See Sec. $G$ of the Supplementary Information for the derivation.) It reads

$\tan \phi\left(\omega, \Phi_{\mathrm{T}}, \Phi_{\mathrm{C}}\right)=\frac{C_{\mathrm{g}}-2 C_{\mathrm{eff}}\left(\Phi_{\mathrm{T}}, \omega\right)}{\sqrt{C_{\mathrm{g}}\left(C_{\mathrm{g}}+4 C_{J}\right)}} \frac{1}{\sqrt{\left(\frac{\omega_{\mathrm{p}}\left(\Phi_{\mathrm{C}}\right)}{\omega}\right)^{2}-1}}$.

In this expression $\omega_{p}\left(\Phi_{C}\right)=1 / \sqrt{L_{J}\left(\Phi_{C}\right)\left(C_{J}+C_{g} / 4\right)}$ is the plasma frequency of the chain, and

$$
C_{\text {eff }}\left(\Phi_{\mathrm{T}}, \omega\right)=C_{1}-C_{J}-\frac{\frac{C_{c}^{2}}{C_{0}-C_{\mathrm{sh}, \mathrm{T}}}\left[\frac{(\hbar \omega)^{2}}{(2 e)^{2}} C_{0}-E_{S}\left(\Phi_{\mathrm{T}}\right)\right]}{\left(C_{0}+C_{\mathrm{sh}, \mathrm{T}}\right) \frac{(\hbar \omega)^{2}}{(2 e)^{2}}-2 E_{S}\left(\Phi_{\mathrm{T}}\right)} .
$$

has dimensions of capacitance. Finally, $E_{S}\left(\Phi_{T}\right)$ is an effective linear inductor energy associated with the Josephson junctions in the transmon, which nonetheless incorporates the transmon non-linearity, and is given by

$E_{S}\left(\Phi_{\mathrm{T}}\right)=E_{J, \mathrm{~T}}\left(\Phi_{\mathrm{T}}\right)-\sqrt{E_{J, \mathrm{~T}}\left(\Phi_{\mathrm{T}}\right) E_{C, \mathrm{~T}}} / 4$.

(See Sec. F of the Supplementary Material for further detail.) The theoretical $\delta \phi\left(\omega, \Phi_{\mathrm{T}}, \Phi_{\mathrm{C}}\right)$ curves plotted in panel a of Fig. 4 were obtained from $\phi\left(\omega, \Phi_{T}, \Phi_{C}\right)$ similarly to Eq. (2) as the difference

$\delta \phi\left(\omega, \Phi_{\mathrm{T}}, \Phi_{\mathrm{C}}\right)=\phi\left(\omega, \Phi_{\mathrm{T}}, \Phi_{\mathrm{C}}\right)-\phi\left(\omega, \Phi_{0} / 2, \Phi_{\mathrm{C}}\right)$.

\section{Analysis of the experimental data}

To extract the relative frequency shift $\delta \phi_{n}\left(\Phi_{T}, \Phi_{C}\right)$ from the data presented in Fig. 2 of the main text, we go about as follows. At a fixed value of the magnetic field that determines $\Phi_{T}$ and $\Phi_{C}$, we fit each of the peaks in the transmission spectrum individually with a Lorentzian. This gives the center frequency of the peaks. From these peak positions, we obtain $\delta \phi_{n}\left(\Phi_{T}, \Phi_{C}\right)$ experimentally using Eq. (1) at a particular $\Phi_{\mathrm{T}}$ and $\Phi_{\mathrm{C}}$. Next we extract the transmon frequency $\omega_{\mathrm{T}}$ for the transmon coupled to the chain from the experimentally determined $\delta \phi_{n}\left(\Phi_{T}, \Phi_{C}\right)$. The details are as follows. Empirically, we find that the experimentally determined $\delta \phi_{n}\left(\Phi_{\mathrm{T}}, \Phi_{\mathrm{C}}\right)$ vs. $\omega_{n}$ data-points fit an arctangent lineshape

$F(\omega)=(1-A)\left(\frac{1}{\pi} \arctan \left(\frac{2\left(\omega-\omega_{\mathrm{T}}\right)}{\Gamma_{\mathrm{T}}}\right)+\frac{1}{2}\right)+A$

very well, for suitable choices of the parameters $A, \omega_{\mathrm{T}}$, and $\Gamma_{\mathrm{T}}$. (In the parameter regime where our device operates, the theoretically predicted phase shift $\delta \phi\left(\omega, \Phi_{\mathrm{T}}, \Phi_{\mathrm{C}}\right)$ also closely approximates this line shape.) We therefore fit the measured $\delta \phi_{n}$ vs. $\omega_{n}$ at fixed $\Phi_{\mathrm{T}}$ and $\Phi_{\mathrm{C}}$ to Eq. (15), interpreting $\omega_{T}$ as the frequency and $\Gamma_{T}$ as the resonance width of the transmon when it is coupled to the chain. Before we can quantitatively compare the experimental results for $\delta \phi_{n}\left(\Phi_{T}, \Phi_{C}\right)$ to the theoretically predicted $\delta \phi\left(\omega, \Phi_{\mathrm{T}}, \Phi_{\mathrm{C}}\right)$ (Eq. (11)), one final model parameter, namely the maximum transmon Josephson energy $E_{J, T, \max }$ must be determined from the experimental data. The general procedure is as follows. Our theoretical model predicts that in the regime where the actual device operates, this transmon frequency is very nearly equal to the isolated $\left(L_{j} \rightarrow \infty\right)$ transmon frequency, i.e., the chain only slightly renormalizes the transmon frequency, and indeed, we see little $\Phi_{C}$ dependence in the extracted $\omega_{\mathrm{T}}$. At $\Phi_{\mathrm{T}}=n \Phi_{0}, n=0, \pm 1, \pm 2, \ldots$, where the transmon Josephson energy is maximal, we therefore use the isolated transmon result (see Sec. F of the Supplementary Information):

$\omega_{\mathrm{T}}\left(\Phi_{\mathrm{T}}=n \Phi_{0}\right)=\sqrt{E_{C, \mathrm{~T}} E_{J, \mathrm{~T}, \max }}-E_{C, \mathrm{~T}} / 8$

Taking the average over $n$ of the experimentally determined $\omega_{T}\left(\Phi_{T}=\right.$ $\left.n \Phi_{0}\right)$, and using our first principle estimate for $E_{C, T}$ in Table 1, we obtain

$\omega_{\mathrm{T}, \max } / 2 \pi \equiv \omega_{\mathrm{T}}\left(\Phi_{\mathrm{T}}=0\right) / 2 \pi=(4.64 \pm 0.01) \mathrm{GHz}$,

$E_{J, \mathrm{~T}, \max } / h=(10.2 \pm 0.4) \mathrm{GHz}$

The theoretical curves in Fig. 4a were then obtained using the system parameters in Table 1 in Eqs. (11), (14), (13), and (4). The full data set covers many transmon periods. Within a given transmon period, we generally analyze data at several values of $\Phi_{\mathrm{T}}$ in the interval from $-0.3 \phi_{0}$ to $0.3 \phi_{0}$. Each transmon period is measured at different $\Phi_{C}$. We also take into account the small variation in $\Phi_{C}$ as the transmon flux sweeps through one flux quantum. The experimental points in Fig. $4 \mathrm{~b}$ are obtained as the transmon width $\Gamma_{\mathrm{T}}$ closest to $\Phi_{\mathrm{T}}=0$. The error bars come from the least square fit using Eq. (15). The theoretical width is obtained from a fit of the phase shift $\delta \phi\left(\omega, \Phi_{\mathrm{T}}, \Phi_{\mathrm{C}}\right)$ with the arctangent of Eq. (15).

\section{Acknowledgements}

The authors would like to thank F. Balestro, D. Dufeu, E. Eyraud, J. Jarreau, J. P. Girard, T. Meunier, and W. Wernsdorfer, for early support with the experimental setup. Very fruitful discussions with C. K. Andersen, H. U. Baranger, D. Basko, S. Bera, J. J. Garcia Ripoll, S. M. Girvin, F. Hekking, K. Le Hur, C. Mueller, A. Parra, and E. Solano are strongly acknowledged. The sample was fabricated in the clean rooms Nanofab and PTA (Upstream Technological Platform).This research was supported by the ANR under contracts CLOUD (project number ANR-16-CE24-0005), GEARED (project number ANR-14-CE26-0018), by the UGA AGIR program, by the National Research Foundation of South Africa (Grant No. 90657), and by the PICS contract FERMICATS. J.P.M. acknowledges support from the Laboratoire $d^{\prime}$ excellence LANEF in Grenoble (ANR-10-LABX-51-01). R.D. and S.L. acknowledge support from the CFM foundation. N.G. acknowledges support from the Fondation Nanosciences de Grenoble. 


\section{DATA AVAILABILITY}

The data that support the findings of this study are available from the corresponding author upon reasonable request.

\section{AUTHOR CONTRIBUTIONS}

J.P.M., S.F., and N.R designed the experiment. J.P.M. and N.R. fabricated the device. J. P.M. and S.L. performed the experiment and analysed the data with help from S.F, N. R., and I.S., while S.F. and I.S. provided the theoretical support. All co-authors participated in setting up the experimental platform, and took part in writing the paper.

\section{ADDITIONAL INFORMATION}

Supplementary information accompanies the paper on the npj Quantum Information website (https://doi.org/10.1038/s41534-018-0104-0).

Competing interests: The authors declare no competing interests.

Publisher's note: Springer Nature remains neutral with regard to jurisdictional claims in published maps and institutional affiliations.

\section{REFERENCES}

1. Logan, D. E. Many-body quantum theory in condensed matter physics-an introduction. J. Phys. A: Math. General. 38, 1829 (2005).

2. Bloch, I., Dalibard, J. \& Zwerger, W. T Many-body physics with ultracold gases. Rev. Mod. Phys. 80, 885-964 (2008).

3. Haroche, S. \& Raimond, J.-M. Exploring the Quantum: Atoms, Cavities, and Photons. (Oxford University Press, Oxford, England, 2006).

4. Hur, K. L. et al. Many-body quantum electrodynamics networks: Non-equilibrium condensed matter physics with light. Comptes Rendus Phys. 17, 808-835 (2016).

5. Houck, A. A., Tiireci, H. E. \& Koch, J. On-chip quantum simulation with superconducting circuits. Nat. Phys. 8, 292-299 (2012).

6. lacopo Carusotto \& Ciuti, C. Quantum fluids of light. Rev. Mod. Phys. 85, 299-366 (2013).

7. Sundaresan, N. M. et al. Beyond strong coupling in a multi-mode cavity. Phys. Rev. $X$ 5, 021035-7 (2015).

8. Wang, C. et al. A Schrodinger cat living in two boxes. Science 352, 1087-1091 (2016).

9. Naik, R. K. et al. Random access quantum information processors using multimode circuit quantum electrodynamics. Nat. Commun. 8, 1904 (2017).

10. Yanbing Liu \& Houck, A. A. Quantum electrodynamics near a photonic bandgap, Nat. Phys., 13, 1-6 (2016).

11. Mirhosseini, M. et al, Superconducting metamaterials for waveguide quantum electrodynamics, arXiv.org, 1802.01708 (2018).

12. Forn-Díaz, P. et al. Ultrastrong coupling of a single artificial atom to an electromagnetic continuum in the nonperturbative regime. Nat. Phys. 13, 39-43 (2017).

13. Magazzu, L. et al. Probing the strongly driven spin-boson model in a superconducting quantum circuit. Nat. Commun. 9, 1403 (2018).

14. Niemczyk, T. et al. Circuit quantum electrodynamics in the ultrastrong-coupling regime. Nat. Phys. 6, 772-776 (2010).

15. Yoshihara, F. et al. Superconducting qubit-oscillator circuit beyond the ultrastrong-coupling regime. Nat. Phys. 13, 44 (2017).

16. Bosman, S. J. et al. Multi-mode ultra-strong coupling in circuit quantum electrodynamics. npj Quantum Inf. 3, 1 (2017).

17. Forn-Diaz P., Lamata L., Rico E., Kono J. \& E. Solano. Ultrastrong coupling regimes of light-matter interaction, arXiv.org, 1804.09275. (2018).

18. Hur, K. L. Kondo resonance of a microwave photon. Phys. Rev. B 85, 140506 (2012).

19. Moshe Goldstein, M. H., Devoret, M., Houzet \& Glazman, L. Inelastic Microwave Photon Scattering off a Quantum Impurity in a Josephson-Junction Array. Phys. Rev. Lett. 110, 017002 (2013).

20. Peropadre, B., Zueco, D., Porras, D. \& Garcia-Ripoll, J. Nonequilibrium and Nonperturbative Dynamics of Ultrastrong Coupling in Open Lines. Phys. Rev. Lett. 111, 243602 (2013).

21. Gheeraert, N., Bera, S. \& Florens, S. Spontaneous emission of Schrodinger cats in a waveguide at ultrastrong coupling. New J. Phys. 19, 023036 (2017).

22. Gheeraert, N. et al, Particle Production in Ultra-Strong Coupling Waveguide QED, arXiv.org, 1802.01665 (2018).

23. Hewson, A. C. The Kondo Problem to Heavy Fermions (Cambridge Studies in Magnetism). Cambridge: Cambridge University Press, https://doi.org/10.1017/ CBO9780511470752 (1993).
24. Plourde, B. L. T., Tang H. W., Rouxinol, F. \& LaHaye M. D. in SPIE Sensing Technology + Applications, (eds Eric Donkor, Andrew R Pirich \& Michael Hayduk) (SPIE, 2015) p $95000 \mathrm{M}$.

25. Rastelli, G. \& Pop, I. M. Tunable ohmic environment using Josephson junction chains. Phys Rev B 97(20), 205429 (2018).

26. Jung, P., Ustinov, A. V. \& Anlage, S. M. Progress in superconducting metamaterials Supercond. Sci. Technol. 27, 073001 (2014).

27. Meiser, D. \& Meystre, P. Superstrong coupling regime of cavity quantum electrodynamics. Phys. Rev. A 74, 065801-4 (2006).

28. Weissl, T. et al. Kerr coefficients of plasma resonances in Josephson junction chains. Phys. Rev. B 92, 104508-10 (2015).

29. Devoret, M. H., Girvin, S. \& Schoelkopf, R. Circuit-QED: How strong can the coupling between a Josephson junction atom and a transmission line resonator be? Ann. der Phys. 16, 767-779 (2007)

30. Peropadre, B., Forn-Diaz, P., Solano, E. \& Garcia-Ripoll, J. J. Switchable Ultrastrong Coupling in Circuit QED. Phys. Rev. Lett. 105, 023601-4 (2010).

31. Bourassa, J. et al. Ultrastrong coupling regime of cavity QED with phase-biased flux qubits. Phys. Rev. A 80, 032109 (2009).

32. Forn-Diaz, P. et al. Observation of the Bloch-Siegert Shift in a Qubit-Oscillator System in the Ultrastrong Coupling Regime. Phys. Rev. Lett. 105, 237001 (2010).

33. Braumüller, J. et al. Analog quantum simulation of the Rabi model in the ultrastrong coupling regime. Nat. Commun. 8, 779 (2017).

34. Langford, N. K. et al. Experimentally simulating the dynamics of quantum light and matter at deep-strong coupling. Nat Commun. 8, 1-9 (2017).

35. Astafiev, O. et al. Resonance fluorescence of a single artificial atom. Science $\mathbf{3 2 7}$ 840-843 (2010).

36. Hoi, I. C., Wilson, C. M., Johansson, G. \& Palomaki, T. Demonstration of a singlephoton router in the microwave regime. Phys. Rev. Lett. 107, 073601 (2011).

37. Haeberlein, M. et al. Spin-boson model with an engineered reservoir in circuit quantum electrodynamics, arXiv.org, 1506.09114vl (2015).

38. Geerligs, L. J., Peters, M., de Groot, L. E. M., Verbruggen, A. \& Mooij, J. E. Charging effects and quantum coherence in regular Josephson junction arrays. Phys. Rev. Lett. 63, 326-329 (1989).

39. Chow, E., Delsing, P. \& Haviland, D. B. Length-scale dependence of the superconductor-to-insulator quantum phase transition in one dimension. Phys. Rev. Lett. 81, 204-207 (1998).

40. Fazio, R. \& van der Zant, H. Quantum phase transitions and vortex dynamics in superconducting networks. Phys. Rep 355, 235-334 (2001).

41. Cedergren, K. et al. Insulating Josephson Junction Chains as Pinned Luttinger Liquids. Phys. Rev. Lett. 119, 167701-5 (2017).

42. Corlevi, S., Guichard, W. T., Hekking, F. W. T. J. \& Haviland, D. B. Phase-charge duality of a Josephson junction in a fluctuating electromagnetic environment. Phys. Rev. Lett. 97, 96802 (2006).

43. Pop, I. M. et al. Measurement of the effect of quantum phase slips in a Josephson junction chain. Nat. Phys. 6, 589-592 (2010).

44. Ergiil, A. et al. Localizing quantum phase slips in one-dimensional josephson junction chains. New J. Phys. 15, 095014 (2013).

45. Likharev, K. K. \& Zorin, A. B. Theory of the Bloch-wave oscillations in small Josephson junctions. J. Low. Temp. Phys. 59, 347-382 (1985).

46. Mooij, J. E. \& Schon, Gerd Propagating plasma mode in thin superconducting filaments. Phys. Rev. Lett. 55, 114-117 (1985).

47. Masluk, N., Pop, I., Kamal, A., Minev, Z. \& Devoret, M. Microwave Characterization of Josephson Junction Arrays: Implementing a Low Loss Superinductance. Phys. Rev. Lett. 109, 137002 (2012).

48. Bell, M., Sadovskyy, I., loffe, L., Kitaev, A. \& Gershenson, M. Quantum Superinductor with Tunable Nonlinearity. Phys. Rev. Lett. 109, 137003 (2012).

49. Altimiras, $C$. et al. Tunable microwave impedance matching to a high impedance source using a Josephson metamaterial. Appl. Phys. Lett. 103, 212601 (2013).

50. Muppalla, P. R. et al. Bistability in a mesoscopic Josephson junction array resonator. Phys. Rev. B 97, 024518-11 (2018).

51. Krupko, Y. et al. Kerr nonlinearity in a superconducting Josephson metamaterial. Phy. Rev. B 98, (2018).

52. Nataf, P. \& Ciuti, C. No-go theorem for superradiant quantum phase transitions in cavity QED and counter-example in circuit QED, Nature. Communications 1, 72 (2010).

53. Garcia-Ripoll, J. J., Peropadre, B. \& De, S. Liberate, Light-matter decoupling and $A^{2}$ term detection in superconducting circuits. Sci. Rep. 5, srepl6055 (2015).

54. Malekakhlagh, M. \& Türeci, H. E. Origin and implications of an $A^{2}$-like contributionin the quantization of circuit-QED systems. Phys. Rev. A 93, 012120-19 (2016).

55. Gely, M. F. et al. Convergence of the multimode quantum Rabi model of circuit quantum electrodynamics. Phys. Rev. B 95, 245115-5 (2017).

56. Andersen, C. K. \& Blais, A. Ultrastrong coupling dynamics with a transmon qubit New J. Phys. 19, 023022 (2017). 
57. Sanchez-Burillo, E., Zueco, D., Garcia-Ripoll, J. J., \& Martin-Moreno, L. Scattering in the ultra-strong regime: nonlinear optics with one photon. Phys. Rev. Lett. 113, 263604-5.

58. Snyman, I. \& Florens, S. Robust Josephson-Kondo screening cloud in circuit quantum electrodynamics. Phys. Rev. B 92, 085131 (2015).

59. Wang, $C$. et al. Surface participation and dielectric loss in superconducting qubits. Appl. Phys. Lett. 107, 162601 (2015).

60. Dial, O. et al. Bulk and surface loss in superconducting transmon qubits. Supercond. Sci. Technol. 29, 044001.

61. Lecocq, F. et al. Junction fabrication by shadow evaporation without a suspended bridge. Nanotechnology 22, 315302 (2011).

62. Devoret, M. H. Quantum fluctuations in electrical circuits, Les Houches Session LXIII (1995).
Open Access This article is licensed under a Creative Commons Attribution 4.0 International License, which permits use, sharing, adaptation, distribution and reproduction in any medium or format, as long as you give appropriate credit to the original author(s) and the source, provide a link to the Creative Commons license, and indicate if changes were made. The images or other third party material in this article are included in the article's Creative Commons license, unless indicated otherwise in a credit line to the material. If material is not included in the article's Creative Commons license and your intended use is not permitted by statutory regulation or exceeds the permitted use, you will need to obtain permission directly from the copyright holder. To view a copy of this license, visit http://creativecommons. org/licenses/by/4.0/.

(c) The Author(s) 2019 